\title{
Recent Advances of Modern Protocol for C-C Bonds-The Suzuki Cross-Coupling
}

\author{
Jadwiga Sołoducho*, Kamila Olech, Agnieszka Świst, Dorota Zając, Joanna Cabaj \\ Faculty of Chemistry, Wrocław University of Technology, Wrocław, Poland \\ Email: ${ }^{*}$ jadwiga.soloducho@pwr.wroc.pl
}

Received April 30, 2013; revised May 30, 2013; accepted June 30, 2013

Copyright (C) 2013 Jadwiga Sołoducho et al. This is an open access article distributed under the Creative Commons Attribution License, which permits unrestricted use, distribution, and reproduction in any medium, provided the original work is properly cited.

\begin{abstract}
Over the past 20 years, small molecule solid phase synthesis has become a powerful tool in the discovery of novel molecular materials. In the development of organic chemistry, the carbon-carbon bond formation has always been one of the most useful and fundamental reaction. The current review summarizes recent developments in metal-catalyzed coupling reactions. The following method is discussed in detail-the cross-coupling of aryl halides with aryl boronic acids (the Suzuki coupling), and the others $\mathrm{C}-\mathrm{C}$ bond formation reactions as the palladium-catalyzed reaction between an aryl and (or) alkyl halide and a vinyl functionality (the Heck reaction); and the palladium-catalyzed cross-coupling reaction of organostannyl reagents with a variety of organic electrophiles (the Stille reaction) - are mentioned.
\end{abstract}

Keywords: C-C Bond Formation; Coupling Reactions; Palladium Catalysts; Suzuki Coupling; Heterocyclic Copolymers

\section{Introduction}

Recently, small-molecule solid-phase synthesis has become a powerful tool in the discovery of new drugs or molecular materials. The methods are investigated by the time in details: the cross-coupling of aryl halides with aryl boronic acids (the Suzuki coupling); the palladiumcatalyzed reaction between an aryl and (or) alkyl halide and a vinyl functionality (the Heck reaction); and the palladium-catalyzed cross-coupling reaction of organostannyl reagents with a variety of organic electrophiles (the Stille reaction) [1].

Organic transformations in aqueous media have received also much attention because water is mainly harmless to the environment. The cross-coupling reaction of alkenyl and aryl halides with organoborane derivatives in the presence of a palladium catalyst and a base ( $\mathrm{Su}-$ zuki reaction) has often been carried out in an organicaqueous mixed solvent. Although Davidson and Triggs [2] discovered in 1968 that arylboronic acids reacted with palladium(II) acetate to give corresponding biaryls, and Garves [3] in 1970 that arylsulfinic acids could be coupled to biaryls using $\mathrm{Pd}(\mathrm{II})$ in aqueous solvents, it was not until 1979 when biaryls could efficiently be prepared by a palladium-catalyzed reaction. Miyaura and Suzuki

"Corresponding author.
[4] reported that cross-coupling reactions between alkenylboranes and organic halides were efficiently catalyzed by a catalytic amount of tetrakis (triphenylphoshine) palladium $\left(\mathrm{Pd}\left(\mathrm{PPh}_{3}\right)_{4}\right)$ in the presence of a suitable base.

In this context, using the conventional bridged derivatives of phenoxazine [5], phenothiazine, fluorene, carbazole as a functional segments, the palladium-catalyzed condensation halide arylenes by Suzuki-, and other coupling reactions were used as well-established procedure in modern organic synthesis [6]. The coupling products find good applications as intermediates in the preparation of materials (Figure 1). However, the Suzuki-type reactions catalyzed by heterogeneous palladium involving bromoarenes are relatively unexplored. In this context, polymer-supported catalysts have become valuable tools offering many advantages such as simplification of product synthesis.

Nowadays, palladium-catalyzed Suzuki-Miyaura coupling reaction $[7,8]$ of aryl halides is one of the most effective methods for the construction of biaryls or substituted aromatic moieties in organic synthesis. Although homogeneous palladium catalysts have been extensively investigated [9-14], their industrial applications remained limited due to the difficulty in the separation process from the products for recycling [9-15]. Heterogenization of the existing homogeneous palladium catalysts offers 


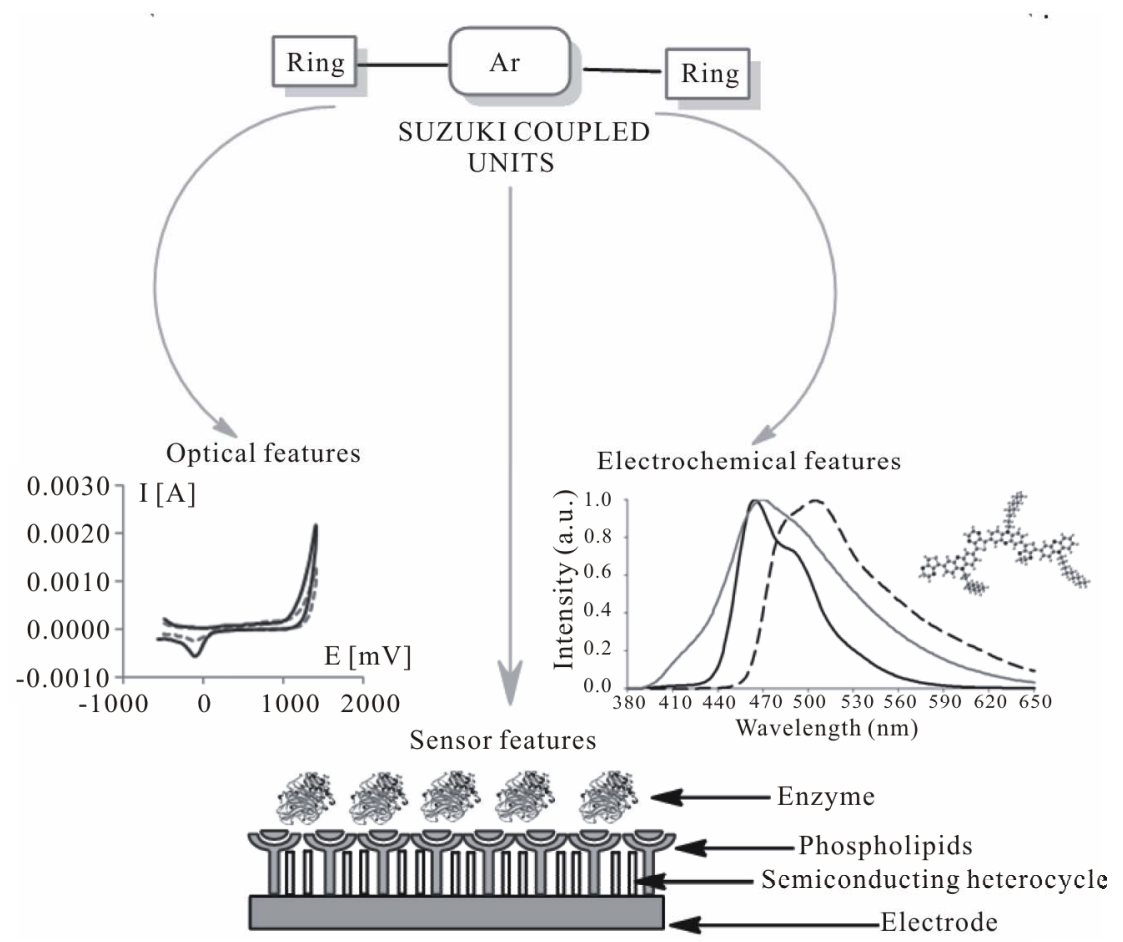

Figure 1. Applications of molecular materials synthesized by Suzuki coupling.

an attractive solution to this problem [16-19]. From a practical point of view, the use of aryl chlorides is highly desirable compared with the more expensive aryl iodides, aryl bromides and aryl triflates.

This short review summarizes the advantages of the Suzuki protocol over other similar cross-coupling reactions, several structurally different ligands that promote the reaction in water [20-23], recent results from ligandless Suzuki procedure, and target molecules mainly produced by the method.

\section{Transition Metals in Synthetic Organic Chemistry}

During the second half of the 20th century, transition metals have come to play an important role in organic chemistry and this has led to the development of a large number of transition metal-catalyzed reactions for creating organic molecules. Transition metals have a unique ability to activate various organic compounds and through this activation they can catalyze the formation of new bonds. One metal that was used early on for catalytic organic transformations was palladium. One event that stimulated research into the use of palladium in organic chemistry was the discovery that ethylene is oxidized to acetaldehyde by air in a palladium-catalyzed reaction and this became the industrially important Wacker process. Subsequent research on palladium-catalyzed carbonylation led to new reactions for the formation of carbon-carbon bonds.
In general, transition metals, and in particular palladium, have been of importance for the development of reactions for the formation of carbon-carbon bonds. In 2005 the Nobel Prize in chemistry was awarded to metal-catalyzed reactions for the formation of carbon-carbon double bonds. The 2010 year the Nobel Prize in chemistry is awarded to the formation of carbon-carbon single bonds through palladium-catalyzed cross-coupling reactions.

\section{Suzuki Coupling}

The Scheme 1 shows the first published Suzuki coupling, which is the palladium-catalyzed cross-coupling between organoboronic acid and halides. Recent catalyst and methods developments have broadened the possible applications enormously, so that the scope of the reaction partners is not restricted to aryls, but includes alkyls, alkenyls and alkynyls. Potassium trifluoroborates and organoboranes or boronate esters may be used in place of boronic acids. Some pseudohalides (for example triflates) may also be used as coupling partners.

\section{Mechanism of the Suzuki Coupling}

In the Suzuki (Scheme 2) as well as Stille mechanism is

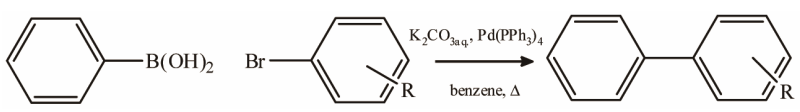

Scheme 1. Typical Suzuki procedure. 
need of activation, and in the Suzuki coupling the boronic acid must be activated, for example with base. The activation of the boron atom features the polarization of the organic ligand, and facilitates transmetalation. If starting materials are substituted with base labile groups (i.e., esters), powdered KF effects this activation while leaving base labile groups unaffected. It is worth to mention, that F- is also advantageous in Stille reaction.

Due to the stability, ease of preparation and low toxicity of the boronic acid compounds, there is currently widespread interest in applications of the Suzuki coupling, with new developments and refinements being reported constantly.

\subsection{Oxidative Addition}

Attaching of the palladium catalyst to the alkyl halide gives rise to the organopalladium complex (Scheme 3). The complex is at the beginning in the cis conformation but isomerizes to the trans conformation. Stereochemistry with vinyl halides are retained but inversion of stereochemistry occurs with allylic or benzylic halides.

\subsection{Transmetalation}

The role of base in the Suzuki coupling is to activate the boron-containing reagent, and also facilitate the forma-

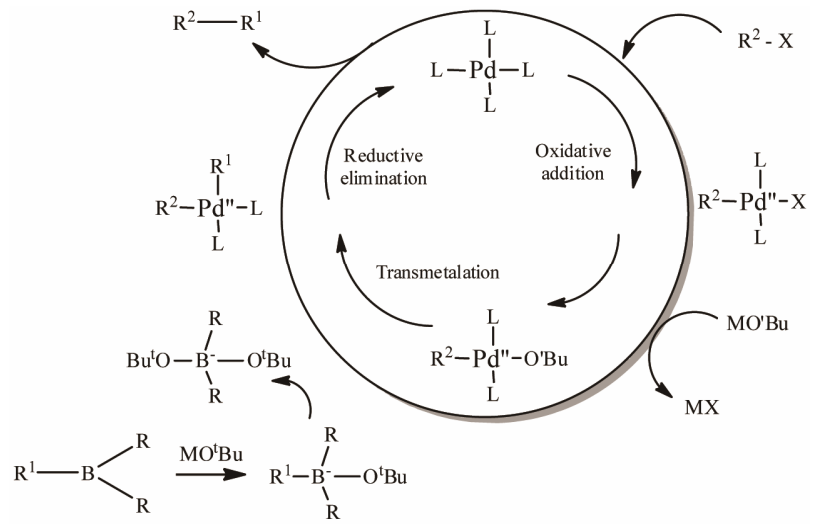

Scheme 2. The mechanism of Suzuki coupling reaction.

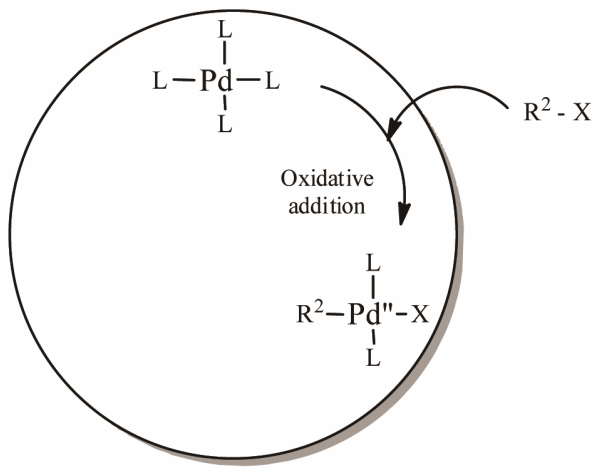

Scheme 3. Oxidative addition of the catalytic cycle. tion of $\mathrm{R}^{1} \mathrm{Pd}-\mathrm{OR}$ from $\mathrm{R}^{1} \mathrm{Pd}-\mathrm{X}$ (Scheme 4). Reaction does not occur in the absence of base. Exact mechanism is unclear.

\subsection{Reductive Elimination}

This final step of catalytic cycle gives the desired product and it also regenerates the palladium catalyst so that it can participate again in the catalytic cycle (Scheme 5). Require the complex to revert back to the cis conformation before reductive elimination can occur.

\section{Suzuki Cross-Coupling as Synthetic Protocol for Branched Heterocyclic Units}

Suzuki reactions are very useful tool for the formation of $\mathrm{Csp}_{2}-\mathrm{Csp} p_{2}$ bonds. This procedure is, however, convenient in the synthesis of conducting polymers. Suzuki reaction is also a powerful method for the formation of $\mathrm{Csp}_{2}-\mathrm{Csp}$ bond. This coupled protocols was also used in our previous work for obtaining branched or hyper-branched sterically crowded heterocyclic structures [24,25] (Scheme 6).

Phenothiazine, as well as phenoxazine, is a wellknown very strong electron donor and has high HOMO energy level because of sulfur atom. Therefore, it was expected phenothiazine ring to be an excellent building block for lowering the ionization potential of conjugated polymers. However, efficient electrogenerated chemiluminescence was observed in co-oligomers of methyl-

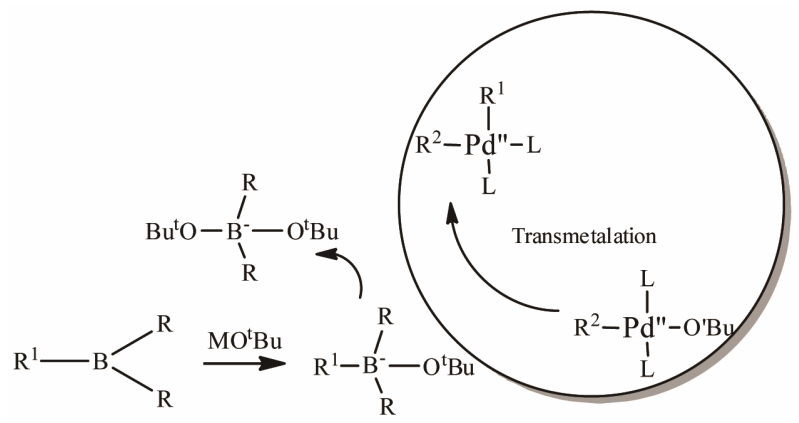

Scheme 4. Transmetalation mechanism.

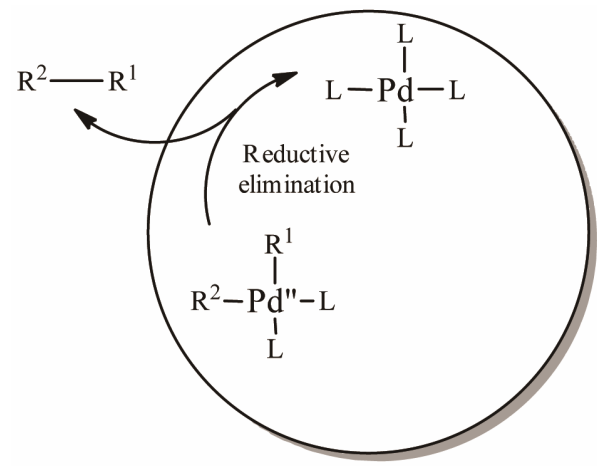

Scheme 5. Reductive elimination. 


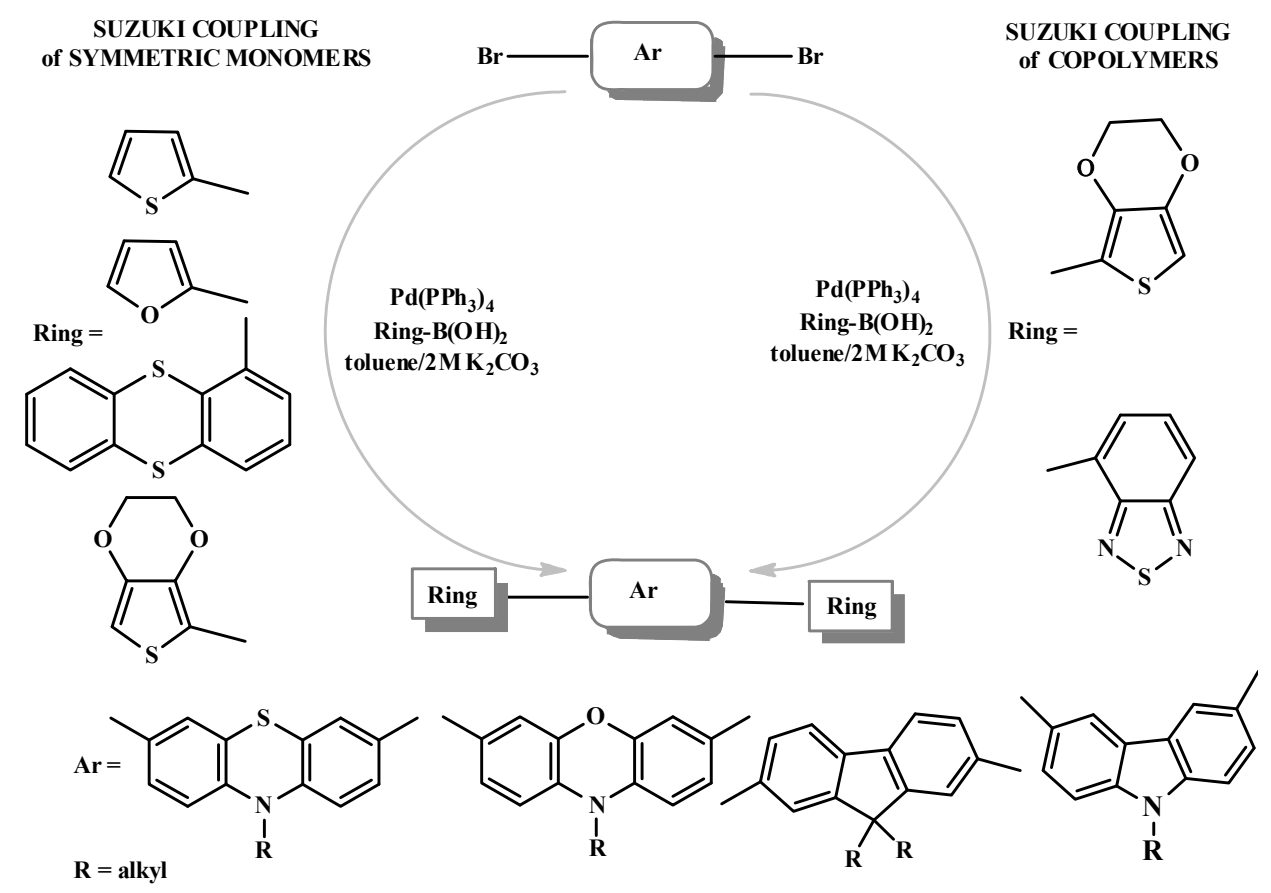

Scheme 6. Pathway of synthesis of symmetric derivatives of arylenes.

phenothiazine and interesting redox properties were found in other oligophenothiazines [26]. Phenothiazines as well as phenoxazines are nonplanar. The possible consequence of the nonplanarity of these rings for the photophysics, light-emitting properties, charge transport of $\pi$-conjugated polymers are motivated. However, the device based on poly(alkylphenothiazine) are not enough to make efficient device since it has unbalanced charge transporting property. In order to improve electron affinity and transporting properties of material we decided to introduced the benzothiadiazole to the oligomer backbone.

Suzuki coupling of dibromoarylenes and boronic acid derivatives was subsequently performed using $\operatorname{Pd}\left(\mathrm{PPh}_{3}\right)_{4}$ as the catalyst. The synthesis of phenothiazine/phenoxazine oligomers was provided as shown in Scheme 7. This type of reactions was carried out in standard conditions [24].

Conversion of $N$-alkyl-3,7-dibromophenoxazine, -phenothiazine to the boronic ester was achieved under reaction conditions in presence 2-isopropoxy-4,4,5,5-tetramethyl1,3,2-dioxaborolane [24]. Alternated benzothiadiazole oligomers were obtained in $50 \%$ yield. The molar ratio of phenoxazine moiety in the oligomers was controlled by adjusting the molar ratio between dibromobenzothiadiazole and monomers ab while a $1 / 1$ molar ratio between the dibromides and the bisborylated compounds was maintained [24].

It was determined that the oligomers were all of rather low molecular weight. Both gave number-average molecular weights of about $3500 \mathrm{~g} / \mathrm{mol}$. This value corre- sponds to a degree of polymerization of seven to eight, meaning that the polymers synthesized here have an average of about 30 aromatic rings per chain [24]. The molecular weights obtained were adequate for processing and film formation, and thus, further optimization of the polymerization was not attempted.

2,1,3-Benzothiadiazole-based oligomers and polymers have been widely studied in recent years as active materials in various optoelectronic devices because of the heterocyclic group and the observed low-band-gap in polymers containing it [27]. Copolymerization of benzothiadiazole with phenoxazine, phenothiazine, fluorene [27] and carbazole [28] or other suitable arylenes can be used as a means to tune the HOMO-LUMO levels in the resulting polymers.

The electron-rich, heterocyclic thianthrene seemed to be a good example for charge transporting materials, since it shows a reversible oxidation behavior at low potential in cyclic voltammetry. The synthesis of thianthrene derivatives (Scheme 8) in moderate yield is shown as follows [25]. The cross-coupling reaction of thianthren-1-yl boronic acid with dibromoalkyl derivatives of fluorene-, carbazole-, diphenylamine-, phenothiazine in presence of catalytic amounts of $\mathrm{Pd}\left(\mathrm{PPh}_{3}\right)_{4}$ in two-phases system at $90^{\circ} \mathrm{C}$ gave a-d, respectively. The obtained semiconducting units as viable luminance and high hole-transporting one, exhibit good solubility in common organic solvents, thermal stability and luminescence in blue region and can be cast into uniform films. They possess good fluorescence quantum [25]. 


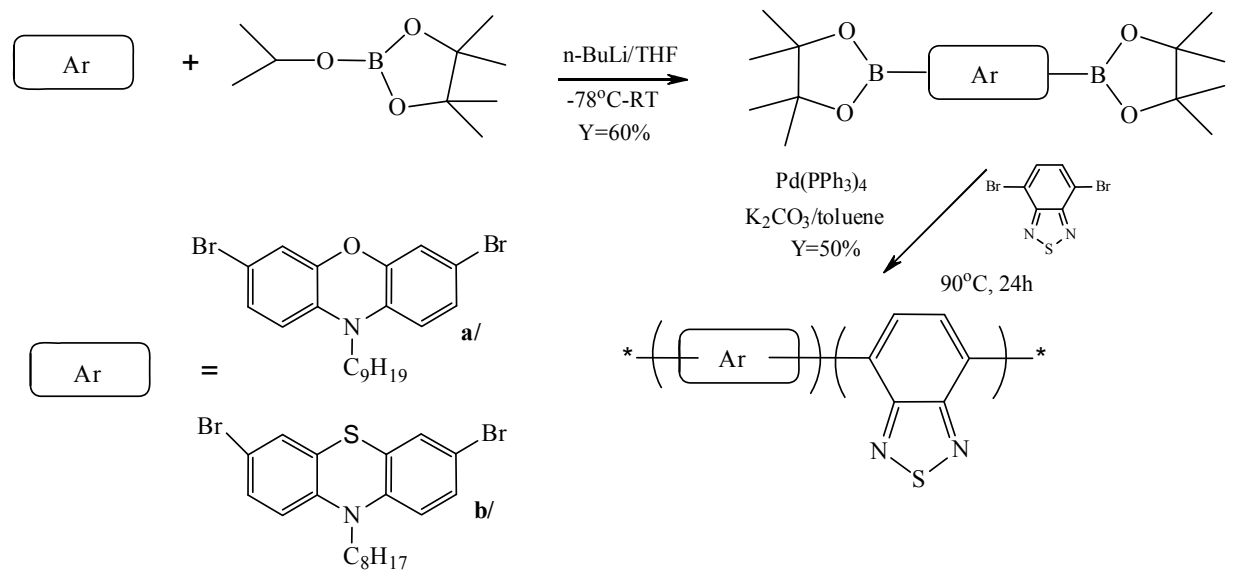

Scheme 7. Synthetic route to the co-oligomers based on benzothiadiazole [by 24].

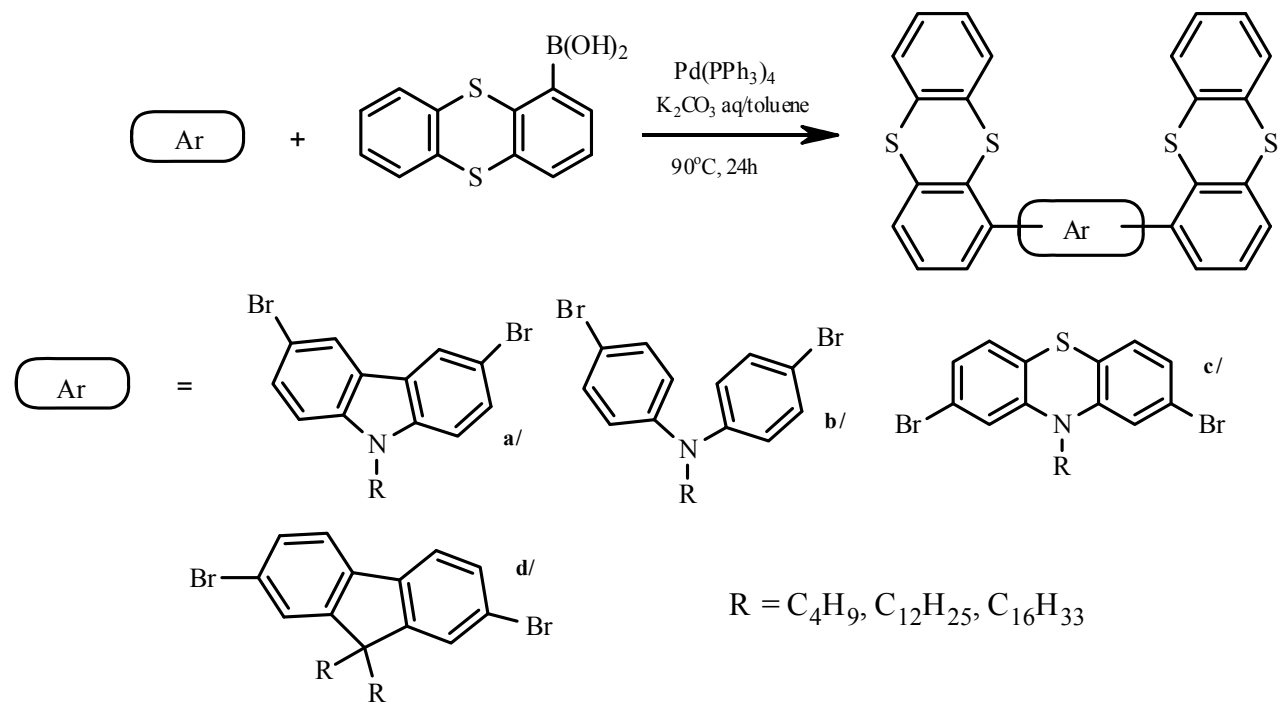

Scheme 8. The synthetic procedure for Suzuki coupled thianthrene derivatives [by 25].

\section{Suzuki Cross-Coupling vs. Other Palladium-Catalyzed Reactions}

In the Suzuki reaction $[8,29]$, the Heck reaction [30], the Kumada reaction [31], the Stille reaction [32], the Negishi reaction [33], and the Sonogashira reaction [34], palladium is known to be particularly effective in activating $s p_{2}$-carbon-halogen bonds even in aqueous media [35]. All reaction types have drawbacks that limit the use in synthesis (Table 1).

Suzuki cross coupling has, on the other hand, less limitations than the other reactions mentioned. In the Heck reaction, for example, where an aryl or vinyl halide and an alkene are converted to a more highly substituted alkene under alladium catalysis, the intermolecular reaction often proceeds well when the alkene is electrophilic. With nucleophilic substituents, the reaction gives less satisfactory effects. The Kumada coupling is very sensitive to air and the presence of radical inhibitors, and this has limited the use of the reaction in aqueous media. In the Stille reaction, stannanes are used as substrates, and many of these are environmentally hazardous. There is no toxicity issue involved in organoborane reagents. For a large-scale setting, a Suzuki coupling is an attractive choice. It is assumed that the electron-donating character of the alkyl groups on the phosphine, as well as its steric bulk, results in a more facile oxidative addition of palladium into the aryl halide. Prior work utilizing aryl chlorides in Suzuki couplings required strongly electronwithdrawing substituents on the aryl chloride.

The organoborane reagents are usually more easy to prepare in the laboratory, and the workup procedures are more simple than using other cross-coupling methods [27-40].

\section{Ligands Suzuki Cross-Coupling}

The vast majority of ligands applied in modern synthesis possess low water solubility. Many strategies have been used to enhance the solubility of metal-ligand complexes 
Table 1. Comparison of palladium catalyzed reactions.

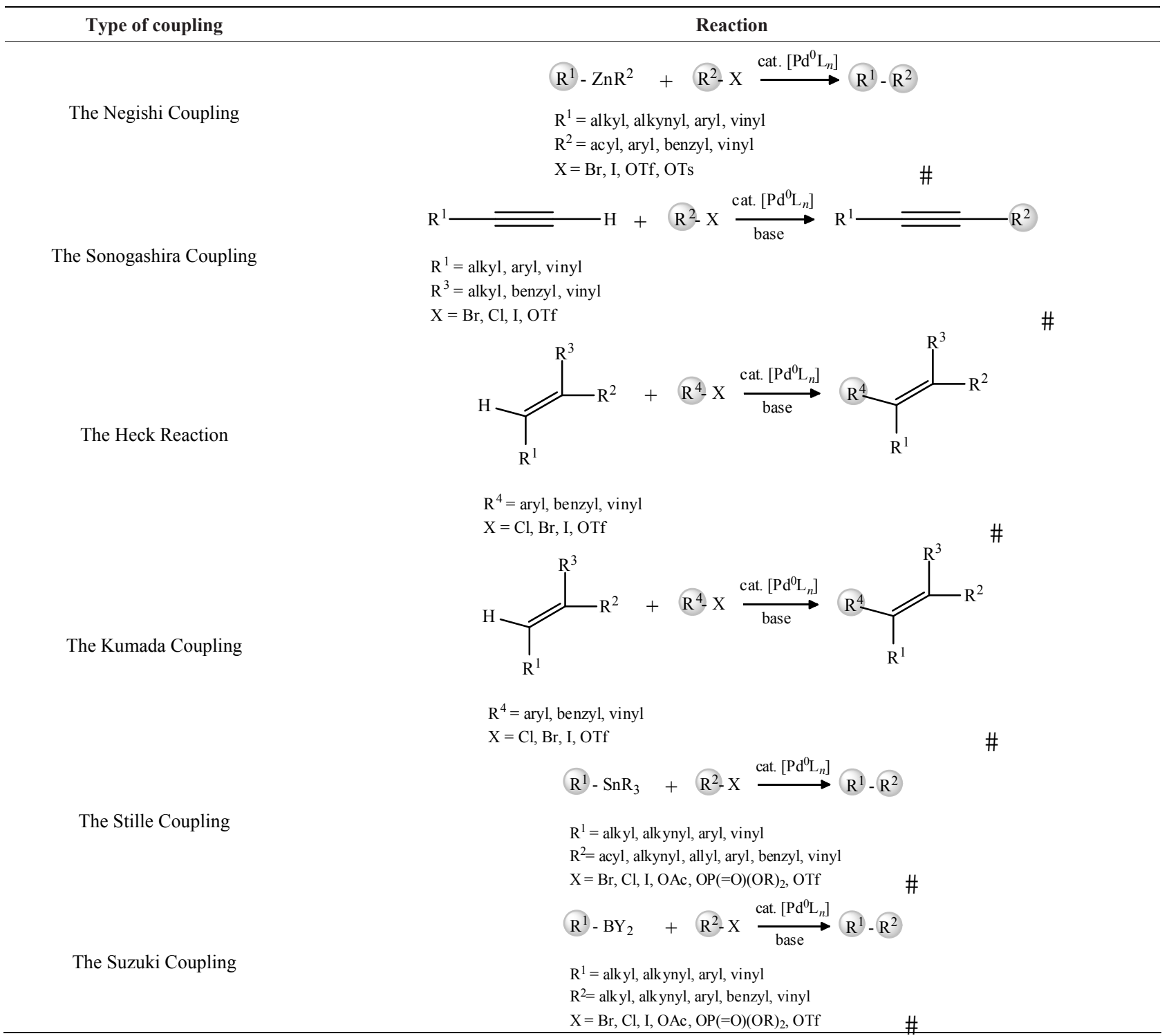

in water of which three are mostly utilized [41]. The catalytic reaction is performed in the presence of micelle-forming surfactants, solubilizing functionalities are added to the ligand, i.e., ionic groups to poorly soluble ligands and easily accessible, water-soluble sources from nature are utilized as ligands.

These include, for example, carbohydrates and the biopolymer chitosan suitable for the Suzuki reaction $[42,43]$ (Scheme 9). Ligands based on monosaccharides usually have only moderate solubility in water and often require incorporation of extra hydrophilic groups to improve the ligand property. Also, a co-solvent or a surfactant is sometimes needed for optimum efficiency. Dissaccharide ligands have the advantage of higher aqueous solubility, but the synthesis of these is usually tedious. Chitosan, on the other hand [44-47], has proven to be a very promising material for organic synthesis and catalysis. For example, Suzuki and Heck reactions have successfully been completed using metal-chitosan complexes as catalysts in aqueous solutions [48]. The complexes have offered both economically and environmentally more favorable conditions for many reactions.

\section{Ligandless Suzuki Cross-Coupling}

From an environmental point of view, development of a new catalytic system without the use of stabilizing phosphine ligands in aqueous media under mild conditions has attracted much attention. The reaction with ligandless catalysts (Table 2) such as $\mathrm{Pd} / \mathrm{C}$ in water has, for example, very recently been investigated $[38,49]$.

Compared to air-sensitive and expensive homogenous palladium catalysts, palladium charcoal can safely be 


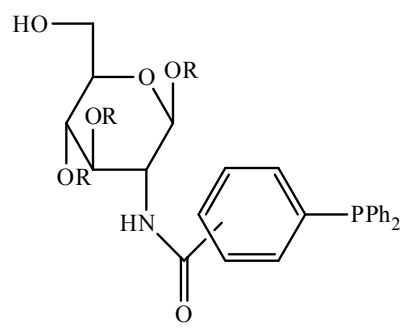

(a)

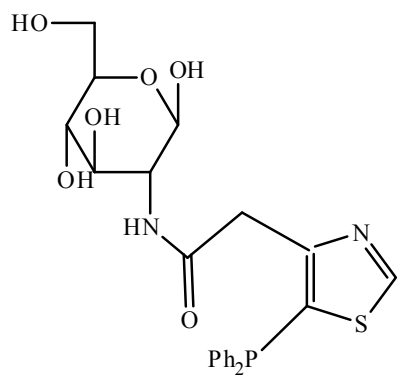

(b)

Scheme 9. Chitosan alternatives suitable for the Suzuki reaction [by 40$]$.

handled and removed from the reaction mixture by simple filtration. The recovered palladium charcoal can be purified and reused as palladium metal. These features are also of great advantage in industrial processes. Other palladium catalysts useful for the ligandless Suzuki reaction in water include $\mathrm{Pd}(\mathrm{OAc})_{2}$ and $\mathrm{PdCl}_{2}[50,51]$.

\section{Targets for the Suzuki Cross-Coupling}

The synthesis of biaryl compounds, reviewed by Stanforth [55], is of importance for numerous agrochemical and pharmaceutical applications. The classic Ullmann methodology [56] is also well-known in this context. Owing, on the other hand, to the versatile chemistry of palladium compounds in carbon-carbon bond-forming reactions, several palladium-catalyzed processes have been proposed as eco-friendly replacement for this stoichiometric protocol. Essentially based on the $\mathrm{Pd}(\mathrm{II})-\mathrm{Pd}(0)$ redox cycle, these processes require in situ regeneration of the active palladium catalyst, which can be achieved, for example, during the Suzuki reaction by various reagents (2-propanol, hydrogen gas, or aqueous alkali salts). In the synthesis of heterocyclic compounds, the aqueous Suzuki reaction has found many applications [57]. A few examples include the structural modifications and preparations of pyrroles [58], indoles [59], pyridines [60], quinoxalines [61], benzofurans [62], pyrimidyl thiazoles [63], pyridyl pyrimidines [63], and imidazoles [64]. The Suzuki reaction of boronic acids in water and a variety of heteroaryl halides has been conducted to prepare 5-substitued heteroaryl pyrimidines, which can be hydrolyzed to 5-substituted uracils as po tential antiviral agents [65]. The reaction could tolerate a broad range of functional groups, including those present in unprotected nucleotides and amino acids. In the preparation of purines on solid phase, Pd catalyses have also successfully been utilized [66]. In the synthesis of polymeric materials (Scheme 7) [24], a hydrocarbon nonmetallic conducting polymer with a rigid rod of benzene rings was synthesized recently in water [67].

\section{Catalytic Asymmetric Suzuki Cross- Coupling}

The asymmetric Suzuki cross-coupling reaction has successfully been accomplished in both organic solvents and inorganic-aqueous mixed solvents. For the preparation of $\mathrm{C}_{2}$-symmetric biaryls, a modified Suzuki cross-coupling method of haloarenes was designed in 1996 by Keay and co-workers [68]. The catalytic asymmetric version of the reaction for the synthesis of axially chiral biaryl compounds was later developed by the Buchwald group [69].

The first, asymmetric Suzuki cross coupling performed in an organic-aqueous mixed solvent resulting in binaphthalene derivatives in up to $63 \%$ ee, was described by Cammidge and Crepy [70] (Scheme 10). In DME, the reaction gave up to $85 \%$ ee. Following these discoveries, other reports on the asymmetric synthesis for chiral binaphhyls or biaryls have been reported, for example, by Castanet et al. [71] in the study on chirality reversal depending on the palladium-chiral phosphine ratio in the reaction of sterically hindered arylboronic acids.

The reaction was performed in DME-water or toluene-EtOH-water. An atropo-enantioselective Suzuki cross coupling towards an axially chiral antimitotic biaryl has very recently been prepared by Herrbach et al. [72] in dioxane-water. Chiral atropisomeric binaphthalenes have also been synthesized in water-DME and a mixture of water-EtOH-toluene.

\section{Optical Properties of Chosen Suzuki Coupled Branched Units}

Benzothiadiazole-based oligomers and polymers have been widely studied in recent years as active materials in various optoelectronic devices because of the heterocyclic group and the observed low band gap in polymers containing it. Copolymerization of benzothiadiazole with phenoxazine, phenothiazine, fluorene and carbazole or other suitable arylenes can be used as a means to tune the HOMO-LUMO levels in the resulting polymers. The HOMO and LUMO energy level of $\pi$-conjugated polymer are important for understanding charge injection processes in the luminescent devices [24].

In flat-panel display applications the full-color capability is required, especially the blue one. Much progress has been made in the development of blue color different 
Table 2. Ligandless catalysts-examples.

Type of coupling References<smiles>Ic1cccc2ccccc12</smiles>

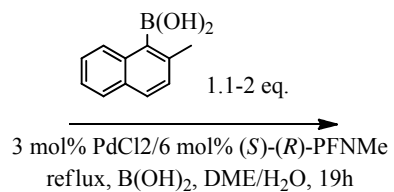<smiles>C[C@H](NCc1ccccc1)[C@@](C)(c1ccccc1)c1ccccc1-c1ccccc1</smiles>

(S)-(R)-PFNMe

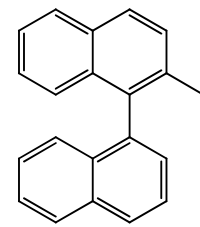

$44 \%, 63 \%$ ee $(R-(-))$
Scheme 10. Asymmetric Suzuki cross coupling [by 40].

OLEDs devices. Many materials have been synthesized and used in blue OLEDs. Moreover, several attempts have been made to stabilize the blue colour via chemical modification, including incorporation of benzothiadiazole, anthracene and other units.

According to the fact, it is worth to mention that the emission spectra of benzothiadiazole-based phenotiazine/phenoxazine derivatives (copolymers synthesized via Suzuki condensation) recorded at different excitation wavelengths in the range $310-390 \mathrm{~nm}$, emitted in the blue region (Figure 2) [24]. Very similar situation we observed in case of thianthrene derivatives of phenothiazine [25].

\section{Thiophene Units}

The class of conducting polymer that has seen significant attention from both academia and industry, largely because of its diverse and often remarkable properties, is polythiophene-based polymers. The introduction of functionalized aryl moieties onto heterocyclic compounds (such as thiophenes) is an important task in organic synthesis. Three main methods exist to get unsymmetrical aryl-aryl coupling: between an aryl halide and an arylboronic acid catalysed by a palladium phosphine complex (Suzuki $[8,29]$ ), between an aryl halide and a Grignard reagent catalysed by a nickel complex (Kumada
[31]), and between an aryl halide and an aryltrialkylstannane catalysed by a palladium complex (Stille [32]).

It is well established that the physical properties of conducting polymers are closely linked to the polymerization conditions and to the structure of their monomeric precursor. Polymerizations of alkylthiophenes can be classified in two categories, i.e. the oxidative electrochemical or chemical polymerization leading to positively charged polymers, and the organometallic couplings leading to neutral polymers [73]. Poly(alkylthiophenes) obtained by the first method are often high-molecular-weight polymers but with a certain amount of irregular couplings [73]. On the other hand polymers obtained by Kumada [31], Stille [32] or Suzuki [8,29] coupling reactions are more regioregular head-to-tail poly(alkylthiophenes) with high molecular weights and conductivities. The drawbacks are the need of stoichiometric amount of metal to functionalize the substrate.

The type and nature of the substituents used to induce solubility and regioregularity in oligo and polythiophenes greatly influence the morphology and band gap in these materials. Several groups have reported the synthesis of well-defined oligomers of thiophenes, especially head-totail coupled ones, to understand the influence of substitution pattern on conjugation and packing. Among the various thiophene based materials reported in the literature, regioregular poly(3-alkylthiophene)s have shown the best promise both in terms of conjugation and device performance [74]. In efforts to identify new classes of thiophene based materials with improved $\pi$-conjugation and charge transport, there is i.e. reported also the synthesis and characterization of a series of structurally defined thiophene based tetramers as model compounds for polymers with thiophene based alkyl solubilizing groups. Three tetramers of thiophene (Scheme 11) bearing alkylthienyl side chains have been synthesized by palladium catalysed Suzuki cross-coupling [75].

One of the other novel thiophene structure obtained by 


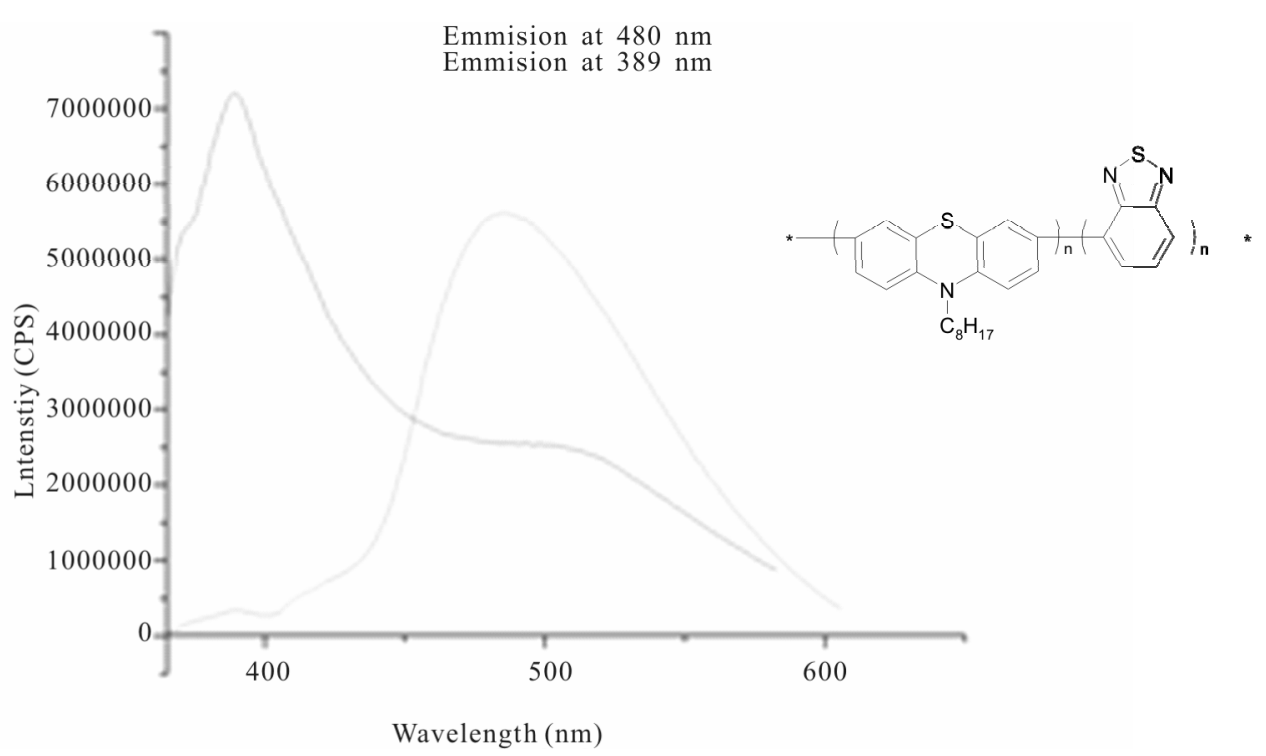

Figure 2. The fluorescence spectra of benzothiadiazole-based phenothiazine copolymer in chloroform solution ( $2 \mu \mathrm{M})$ [by 24].

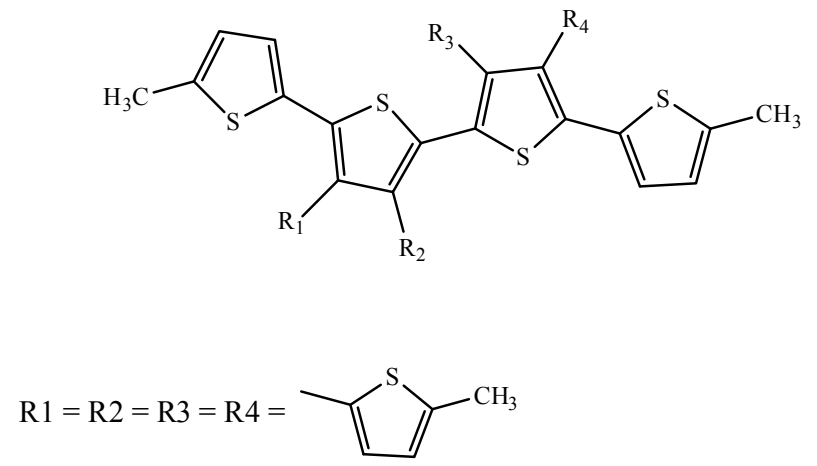

Scheme 11. Structures of substituted thiophene-based tetramers [75].

Suzuki coupling is also the substituted thieno[3,2-b] thiophene molecules with triphenylamine core. The synthetic method allowed the preparation of trisubstituted triphenylamine derivatives of thieno[3,2-b] thiophene and thiophene (Scheme 12) in a combinatorial manner starting from tris (4-bromophenyl) amine in relatively good yields [76]. The newly suggested molecules exhibited extended $\pi$-conjugation with high molar extinction coefficients and some of them demonstrated molecular glass behavior.

It is need to mention that, the pure polythiophenes are generally less suitable for polymerization by the Suzuki-Miyaura coupling method. The reason for this is the electron-rich nature of thiophenes, which slows down the oxidative addition step, resulting in an overall slower reaction and more pronounced deborylation. Deborylation is the main side reaction of the Suzuki-Miyaura coupling, resulting in low molecular weight products. For less electronegative aryl monomers like polyfluorenes,

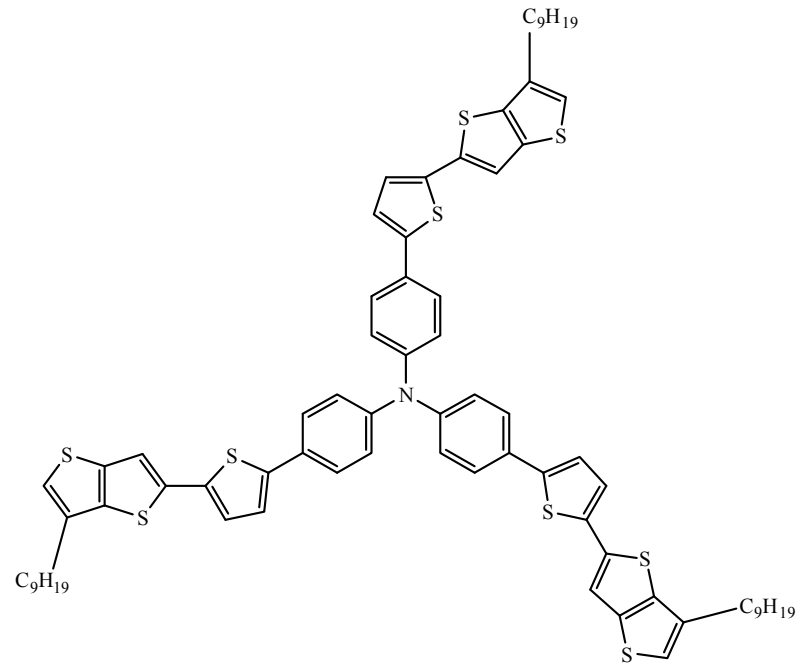

Scheme 12. Star-shaped thiophene derivative [76].

the Suzuki-Miyaura coupling is generally accepted as a better alternative than the Stille coupling, giving higher molecular weight products.

\section{Supramolecular Structures Built of $\pi$-Stacking Thiophene Derivatives}

The development of the methods to create nanostructures has been inspired primarily by natural environment, which displays a wide variety of complex nano-sized structures with great precision. These nano-sized structures in biological systems are specifically put together from two or more small molecular components by means of secondary interactions.

Oligo- and polythiophenes form an important class of conducting materials that can find possible applications 
in, for example, thin film transistors or light-emitting diodes [77-79].

Previous investigations of the bulk properties of the i.e. bis(urea)-substituted thiophene derivatives under consideration have shown that the molecules form one-dimensional fibers in solution in which the thiophene moieties are $\pi$-stacked [80]. Pulse-radiolysis time-resolved microwave conductivity experiments i.e. have also demonstrated that this arrangement provides an efficient path for charge transport within these self-assembled fibers. In fact, it has been shown that, by inducing a high degree of molecular order in thin films of oligothiophenes, it is possible to enhance the mobility of charge carriers [81].

The effects of the chemical composition of conjugated polymers on the supramolecular organization, i.e., the morphology of the active layer, are of major concern to further optimize the performance of the devices. This feature can sufficiently be illustrated with poly(3-alkylthiophene)s.

Since polymerization procedures for regioregular poly(3-substituted-thiophene)s have improved considerably, it is obvious that many functional features are more pronounced for the regioregular material, being either head-to-tail [82] or head-to-head/tail-to-tail, [83] due to an improved ordering of the planar conformation compared to the regioirregular polythiophene.

The latter polymer, without defined interchain interactions, gave rise to low charge-carrier mobilities $\left(10^{-5}\right.$ $\left.\mathrm{cm}^{2} / \mathrm{V} \cdot \mathrm{s}\right)$, whereas the former, with lamellar arrays of polythiophene rods, gave significant higher mobilities $\left(0.01-0.1 \mathrm{~cm}^{2} / \mathrm{V} \cdot \mathrm{s}\right)[84]$.

However, the manufacturing process of thiophene thin film may cause many problems. For instance, vacuum vapor deposition requires much energy and expensive equipment. Although thin film fabrication method via simple wet process have been developed using a polymer solution, it is difficult to obtain polymer thin films with high crystallinity. Ikeda and co-worker overcame these problems and found a facile manufacturing method of thiophene nanosheets with high crystallinity in the solution [85]. They discovered that an alternating copolymer, in which a thiophene derivative and flexible ethylene glycol chain are alternately connected, is folded in some organic solvents in such a way that the thiophene units are stacked each other, and the folded copolymers self-assemble into a 2-dimensional sheet structure (Scheme 13).

The arrangement of the thiophene units in the nanosheet was confirmed to be the same as that manufactured by vacuum vapor-deposition of low-molecularweight thiophene compounds. Therefore, our thiophene nanosheets are feasible to the application of organic electronics devices [85].

These tailor-made supramolecular assemblies will enormously influence the macroscopic properties. The

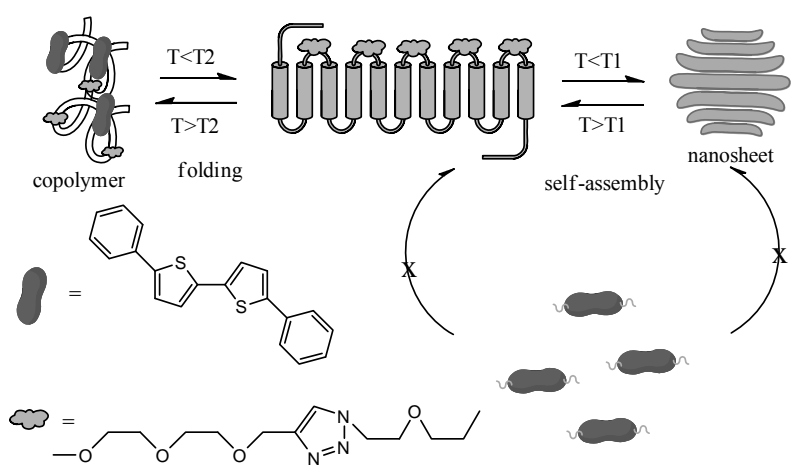

Scheme 13. Concept of Ikada et al. of the supramolecular thiophene nanosheet formation.

combination of supramolecular architecture with functionality in macromolecules will not only give rise to emerging opportunities in materials science, but also significantly contribute to bridge the gap between natural and artificial systems in an effort to fully understand the guidelines used to assemble natural units in the different hierarchies of organization.

\section{Concluding Remarks}

Carbon-carbon bond formations reactions on solid-support are highly useful and versatile methods needed for the development of modern organic synthesis discovery.

The review focuses on the investigation of the solidphase adaption of the Suzuki, the coupling reactions due the fact that these have proven to be both reliable and efficient methods.

Although the method has found many applications in synthesizing different molecules, there is still much work on the development towards an efficient catalyst applicable for structurally different substrates. The work on developing a catalyst working entirely in water has resulted in a few remarkable discoveries. However, during the next few years, it is anticipated that new and more efficient catalysts will be discovered, giving even more excellent results in aqueous media.

Since the first reports by Miyaura and Suzuki in 1979, the palladium-catalyzed cross-coupling reactions of organoboron derivatives with organic electrophiles have been widely employed in both academic and industrial laboratories and have significantly contributed to the efficient synthesis also on a large scale of a wide range of interesting compounds including pharmaceuticals, agrochemicals and synthetic intermediates.

In spite of the fact that, in recent years, many new transition metal-catalyzed methods allowing $\mathrm{C}-\mathrm{C}$ bond formation have been developed, the Suzuki-Miyaura reaction is still one of the most reliable and environmentally friendly tools for installing a wide range of nonfunctionalized and functionalized carbon substitu- 
ents on (hetero)aromatic systems with exquisite chemoand site-selectivity and is by far the most versatile method for the synthesis of industrially relevant functionalized heteroarenes and unsymmetrical biheteroaryl derivatives.

Finally, summarizing the results illustrated in this paper, it may be noted that for substrates bearing different halogen atoms chemoselective cross-couplings have been accomplished on the basis of the reactivity difference between the halogens. However, the hardest acquirable selectivity in Suzuki-Miyaura monocoupling reactions involving polyhalogenated heteroarenes bearing identical halogen atoms has been shown to be dominated by steric and electronic effects and the presence of directing groups at positions neighboring the reaction sites. Moreover, for symmetrically substituted dihalogenated heteroarenes bearing identical halogen atoms its achievement has sometimes required a careful optimization of the reaction parameters including the nature of the catalyst precursor, the base, the solvent and the molar ratio between electrophile and organoboron reagent.

Interestingly, the efficiency and selectivity of several monoarylation reactions illustrated in the sections of this review have frequently allowed the development of one-pot polycoupling procedures, but, unfortunately, this synthetically useful approach to polysubstituted heteroarenes has not been applied so far to the synthesis of important pharmacologically active agents and structurally complex natural products.

It is believed that many of the one-pot processes developed so far, which are valuable for laboratory syntheses on a small scale, require further studies aimed to make them suitable for large-scale preparations through improvement of their yields and selectivity and development of more efficient and cost effective catalyst systems.

\section{Acknowledgements}

The authors are gratefully acknowledged for the financial support of the Polish National Centre of Progress of Explorations Grant no. NR05-0017-10/2010 and Wroclaw University of Technology.

\section{REFERENCES}

[1] R. Franzen, "The Suzuki, the Heck, and the Stille Reaction/Three Versatile Methods for the Introduction of New C-C Bonds on Solid Support," Canadian Journal of Chemistry, Vol. 78, No. 7, 2000, pp. 957-962. doi:10.1139/v00-089

[2] J. M. Davidson and C. J. Triggs, "Reaction of Metal Ion Complexes with Hydrocarbons. I. Palladation and Some Other New Electrophilic Substitution Reactions. Preparation of Palladium(I)," Journal of the Chemical Society A:
Inorganic, Physical, Theoretical, Vol. 6, 1968, pp. 13241331. doi:10.1039/j19680001324

[3] K. Garves, "Coupling, Carbonylation, and Vinylation Reactions of Aromatic Sulfinic Acids via Organopalladium Intermediates," The Journal of Organic Chemistry, Vol. 35, No. 10, 1970, pp. 3273-3275. doi:10.1021/jo00835a021

[4] N. Miyaura and A. Suzuki, "Stereoselective Synthesis of Arylated (E)-Alkenes by the Reaction of Alk-1-enylboranes with Aryl Halides in the Presence of Palladium Catalyst," Journal of the Chemical Society, Chemical Communications, Vol. 19, 1979, pp. 866-867. doi: $10.1039 / \mathrm{c} 39790000866$

[5] A. Nowakowska-Oleksy, J. Sołoducho and J. Cabaj, "Phenoxazine Based Units-Synthesis, Photophysics and Electrochemistry," Journal of Fluorescence, Vol. 21, No. 1, 2011, pp. 169-178. doi:10.1007/s10895-010-0701-6

[6] J. Sołoducho, J. Cabaj, K. Idzik, A. Nowakowska-Oleksy, A. Świst and M. Lapkowski, "Synthesis, Structure and Properties of Crowded Symmetric Arylenes," Current Organic Chemistry, Vol. 14, No. 12, 2010, pp. 12341244. doi:10.2174/138527210791330468

[7] N. Miyaura, K. Yamada and A. Suzuki, "A New Stereospecific Cross-Coupling by the Palladium-Catalyzed Reaction of 1-Alkenylboranes with 1-Alkenyl or 1-Alkynyl Halides," Tetrahedron Letters, Vol. 20, No. 36, 1979, pp. 3437-3440. doi:10.1016/S0040-4039(01)95429-2

[8] N. Miyaura and A. Suzuki, "Palladium-Catalyzed CrossCoupling Reactions of Organoboron Compounds," Chemical Reviews, Vol. 95, No. 7, 1995, pp. 2457-2483. doi:10.1021/cr00039a007

[9] G. Altenhoff, R. Goddard, C. W. Lehmann and F. Glorius, "A N-Heterocyclic Carbene Ligand with Flexible Steric Bulk Allows Suzuki Cross-Coupling of Sterically Hindered Aryl Chlorides at Room Temperature," Angewandte Chemie International Edition, Vol. 42, No. 31, 2003, pp. 3690-3693. doi:10.1002/anie.200351325

[10] W. A. Herrmann, K. Fele, S. K. Schneider, E. Herdtweck and S. D. Hoffmann, "A Carbocyclic Carbene as an Efficient Catalyst Ligand for C-C Coupling Reactions," Angewandte Chemie International Edition, Vol. 45, No. 23, 2006, pp. 3859-3862. doi:10.1002/anie.200503589

[11] W. J. Tang, A. G. Capacci, X. D. Wei, W. J. Li, A. White, N. D. Patel, J. Savoie, J. J. Gao, S. Rodriguez, B. Qu, N. Haddad, B. Z. Lu, D. Krishnamurthy, N. K. Yee and C. H. Senanayake, "A General and Special Catalyst for SuzukiMiyaura Coupling Processes," Angewandte Chemie, Vol. 122, No. 34, 2010, pp. 6015-6019.

[12] D. H. Lee and M. J. Jin, "An Extremely Active and General Catalyst for Suzuki Coupling Reaction of Unreactive Aryl Chlorides" Organic Letters, Vol. 13, No. 2, 2011, pp. 252-255. doi:10.1021/ol102677r

[13] M. Thimmaiah and S. Fang, "Efficient Palladium-Catalyzed Suzuki-Miyaura Coupling of Aryl Chlorides with Arylboronic Acids Using Benzoferrocenyl Phosphines as Supporting Ligands," Tetrahedron, Vol. 63, No. 29, 2007, pp. 6879-6886. doi:10.1016/j.tet.2007.04.057

[14] Y. Uozumi, Y. Matsuura, T. Arakawa and Y. M. A. Ya- 
mada, "Asymmetric Suzuki-Miyaura Coupling in Water with a Chiral Palladium Catalyst Supported on an Amphiphilic Resin" Angewandte Chemie International Edition, Vol. 48, No. 15, 2009, pp. 2708-2710. doi:10.1002/anie.200900469

[15] D. J. M. Snelders, G. van Koten and R. Gebbink, "Hexacationic Dendriphos Ligands in the Pd-Catalyzed Suzuki-Miyaura Cross-Coupling Reaction: Scope and Mechanistic Studies," Journal of the American Chemical Society, Vol. 131, No. 32, 2009, pp. 11407-11416. doi:10.1021/ja904042h

[16] L. Yin and J. Liebscher, "Carbon-Carbon Coupling Reactions Catalyzed by Heterogeneous Palladium Catalysts," Chemical Reviews, Vol. 107, No. 1, 2006, pp. 133-173. doi:10.1021/cr0505674

[17] M. Lysen and K. Kohler, "Suzuki-Miyaura Cross-Coupling of Aryl Chlorides in Water Using Ligandless Palladium on Activated Carbon," Synlett, Vol. 11, 2005, pp. 1671-1674. doi:10.1055/s-2005-869877

[18] M. Lysen and K. Kohler, "Palladium on Activated Carbon-A Recyclable Catalyst for Suzuki-Miyaura CrossCoupling of Aryl Chlorides in Water," Synthesis, Vol. 4, 2006, pp. 692-698. doi:10.1055/s-2006-926305

[19] N. Gürbüz, I. Özdemir, T. Seçkin, B. Çetinkaya, "Surface Modification of Inorganic Oxide Particles with a Carbene Complex of Palladium: A Recyclable Catalyst for the Suzuki Reaction," Journal of Inorganic and Organometallic Polymers, Vol. 14, 2No. 2, 004, pp.149-159. doi:10.1023/B:JOIP.0000028092.46608.ac

[20] J. Han, Y. Liu and R. Guo, "Facile Synthesis of Highly Stable Gold Nanoparticles and Their Unexpected Excellent Catalytic Activity for Suzuki-Miyaura Cross-Coupling Reaction in Water," Journal of the American Chemical Society, Vol. 131, No. 6, 2009, pp. 2060-2061. doi:10.1021/ja808935n

[21] B. Z. Yuan, Y. Y. Pan, Y. W. Li, B. L. Yin and H. F. Jiang, "A Highly Active Heterogeneous Palladium Catalyst for the Suzuki-Miyaura and Ullmann Coupling Reactions of Aryl Chlorides in Aqueous Media," Angewandte Chemie International Edition, Vol. 49, No. 24, 2010, pp. 4054-4058. doi:10.1002/anie.201000576

[22] M. J. Jin and D. H. Lee, “A practical Heterogeneous Catalyst for the Suzuki, Sonogashira, and Stille Coupling Reactions of Unreactive Aryl Chlorides," Angewandte Chemie International Edition, Vol. 49, No. 6, 2010, pp. 1119-1122. doi:10.1002/anie.200905626

[23] B. Li, Z. Guan, W. Wang, X. Yang, J. Hu, B. Tan and T. Li, "Highly Dispersed pd Catalyst Locked in Knitting Aryl Network Polymers for Suzuki-Miyaura Coupling Reactions of Aryl Chlorides in Aqueous Media," Advanced Materials, Vol. 24, 2No. 25, 012, pp. 3390-3395. doi:10.1002/adma.201200804

[24] A. Nowakowska-Oleksy, J. Cabaj, K. Olech and J. Sołoducho, "Comparative Study of Alternating Low-BandGap Benzothiadiazole Co-Oligomers," Journal of Fluorescence, Vol. 21, No. 4, 2011, pp. 1625-1633. doi:10.1007/s10895-011-0851-1

[25] A. Świst, J. Sołoducho, P. Data and M. Łapkowski, "Thianthrene-Based Oligomers as Hole Transporting
Materials," ARKIVOC, Vol. 2012, No. 3, 2012, pp. 193209. doi:10.3998/ark.5550190.0013.315

[26] M. Sailer, R. A. Gropeanu and T. J. Müller, "Practical Synthesis of Iodo Phenothiazines. A Facile Access to Electrophore Building Blocks," The Journal of Organic Chemistry, Vol. 68, No. 19, 2003, pp. 7509-7512. doi:10.1021/jo034555z

[27] P. Herguth, X. Jiang, M. S. Liu and A. K. Y. Jen, "Highly Efficient Fluorene- and Benzothiadiazole-Based Conjugated Copolymers for Polymer Light-Emitting Diodes," Macromolecules, Vol. 35, No. 16, 2002, pp. 6094-6100. doi: $10.1021 / \mathrm{ma} 020405 \mathrm{z}$

[28] Y. Zhu, R. D. Champion and S. A. Jenekhe, "Conjugated Donor-Acceptor Copolymer Semiconductors with Larg Intramolecular Charge Transfer: Synthesis, Optical Properties, Electrochemistry, and Field Effect Carrier Mobility of Thienopyrazine Based Copolymers," Macromolecules, Vol. 39, No. 25, 2006, pp. 8712-8719.

doi: $10.1021 / \mathrm{ma} 061861 \mathrm{~g}$

[29] N. Miyaura and A. Suzuki, "Stereoselective Synthesis of Arylated (E)-Alkenes by the Reaction of Alk-1-Enylboranes with Aryl Halides in the Presence of Palladium Catalyst," Journal of the Chemical Society, Chemical Communications, Vol. 19, 1979, pp. 866-867. doi:10.1039/c39790000866

[30] R. F. Heck, "Palladium-Catalysed Vinylation of Organic Halides," Organic Reactions, Vol. 27, 1982, pp. 345-390.

[31] T. Hayashi and M. Kumada, "Asymmetric Synthesis Catalyzed by Transition-Metal Complexes with Func- tionalized Chiral Ferrocenylphosphine Ligands," Ac- counts of Chemical Research, Vol. 15, No. 12, 1982, pp. 395-401. doi:10.1021/ar00084a003

[32] J. W. Labadie, D. Tueting and S. K. Stille, "Synthetic Utility of the Palladium-Catalyzed Coupling Reaction of Acid Chlorides with Organotins," The Journal of Organic Chemistry, Vol. 48, No. 24, 1983, pp. 4634-4642. doi: $10.1021 /$ jo00172a038

[33] E. Negishi, "Palladium- or Nickel-Catalyzed cross Coupling. A New Selective Method for Carbon-Carbon Bond Formation," Accounts of Chemical Research, Vol. 15, No. 11, 1982, pp. 340-348. doi:10.1021/ar00083a001

[34] K. Sonogashira, "Development of $\mathrm{Pd}-\mathrm{Cu}$ Catalyzed Cross-Coupling of Terminal Acetylenes with $\mathrm{sp}^{2}$-Carbon Halides," Journal of Organometallic Chemistry, Vol. 653, No. 1-2, 2002, pp. 46-49. doi:10.1016/S0022-328X(02)01158-0

[35] J. Tsuji, "Transition metal reagents and catalysts: Innovations in organic synthesis," John Wiley and Sons, New York, 2002. doi:10.1002/0470854766M. F. Lipton, M. A. Mauragis, M. T. Maloney, M. F. Veley, D. W. Vander-Bor, J. J. Newby, R. B. Appell and E. D. Daugs, "The Synthesis of OSU 6162: Efficient, Lar- ge-Scale Implementation of a Suzuki Coupling," Organic Process Research and Development, Vol. 7, No. 3, 2003, pp. 385-392. doi:10.1021/op025620u

[36] M. Beller and A. Zapf, "Handbook of Organopalladium Chemistry for Organic Synthesis," Wiley, Hoboken, 2002.

[37] D. S. Ennis, J. McManus, W. Wood-Kaczmar, J. Richard- 
son, G. E. Smith and A. Carstairs, "Multikilogram-Scale Synthesis at a Biphenyl Carboxylic Acid Derivative Using a Pd/C-Mediated Suzuki Coupling Approach," Organic Process Research and Development, Vol. 3, No. 4, 1999, pp. 248-252. doi:10.1021/op980079g

[38] Y. Mori, M. Nakamura, T. Wakabayashi, K. Mori and S. Kobayashi, "Efficient Total Synthesis of Khafrefungin: Convergent Approach Using Suzuki Coupling under Thallium-Free Conditions toward Multigram-Scale Synthesis," Synlett, Vol. 4, 2002, pp. 601-603. doi: $10.1055 / \mathrm{s}-2002-22726$

[39] R. Franzén and Y. Xu, "Review on Green Chemistry -Suzuki Cross-Coupling in Aqueous Media," Canadian Journal of Chemistry, Vol. 83, No. 3, 2005, pp. 266-272. doi: $10.1139 / \mathrm{v} 05-048$

[40] A. Herrmann and C. W. Kohlpaintner, "Water-Soluble Ligands, Metal Complexes, and Catalysts: Synergism of Homogeneous and Heterogeneous Catalysis," Angewandte Chemie International Edition in English, Vol. 32, No. 11, 1993, pp. 1524-1544. doi:10.1002/anie.199315241

[41] U. M. Lindström, "Stereoselective Organic Reactions in Water," Chemical Reviews, Vol. 102, No. 8, 2002, pp. 2751-2772.

[42] M. Bellar, J. G. E. Krauter and A. Zapf, "Kohlenhydrat-substituierte Triarylphosphane-Eine Neue Ligandenklasse für die Zweiphasen-Katalyse," Angewandte Chemie International Edition in English, Vol. 36, No. , 1997, pp. 772-774.

[43] J. Zhang and C.-G. Xia, "Kinetic Study of Dichlorocyclopropanation of 4-Vinyl-1-cyclohexene by a Novel Multisite Phase Transfer Catalyst," Journal of Molecular Catalysis A: Chemical, Vol. 206, No. 1-2, 2003, pp. 5968.

[44] T. Vincent and E. Guibal, "Chitosan-Supported Palladium Catalyst. Synthesis Procedure," Industrial and Engineering Chemistry Research, Vol. 41, No. 21, 2002, pp. 51585164. doi:10.1021/ie 0201462

[45] F. Quignard, A. Choplin and A. Damard, "Chitosan: A Natural Polymeric Support of Catalysts for the Synthesis of Fine Chemicals," Langmuir, Vol. 16, No. 24, 2000, pp. 9106-9108. doi:10.1021/la000937d

[46] M.-A. Yin, G.-L. Yuan, Y.-Q. Wu, M.-Y. Huang and Y.-Y. Jiang, "Asymmetric Hydrogenation of Ketones Catalyzed by a Silica-Supported Chitosan-Palladium Complex," Journal of Molecular Catalysis A: Chemical, Vol. 147, No. 1-2, 1999, pp. 93-98. doi:10.1016/S1381-1169(99)00133-8

[47] J. J. E. Hardy, S. Hubert, D. C. Macquarrie and A. J. Wilson, "Chitosan-Based Palladium Catalysts in the Heck and Suzuki Reactions," Green Chemistry, Vol. 6, 2004, p. 53. doi:10.1039/b312145n

[48] H. Sakurai, T. Tsukuda and T. Hirao, " $\mathrm{Pd} / \mathrm{C}$ as a Reusable Catalyst for the Coupling Reaction of Halophenols and Arylboronic Acids in Aqueous Media," Journal of Organic Chemistry, Vol. 67, No. 8, 2002, pp. 2721-2722. doi:10.1021/jo016342k

[49] M. T. Reetz and E. Westermann, "Phosphanfreie Palladium-katalysierte Kupplungen: Die Entscheidende Rolle von Pd-Nanoteilchen," Angewandte Chemie, Vol. 112,
No. 1, 2000, pp. 170-173.

doi:10.1002/(SICI)1521-3757(20000103)112:1<170::AID -ANGE170>3.0.CO;2-A

[50] G. W. Kabalka, V. Namboodiri and L. Wang, "Suzuki Coupling with Ligandless Palladium and Potassium Fluoride," Chemical Communications, Vol. 8, 2001, p. 775. doi:10.1039/b101470f

[51] T. N. Glasnov, S. Findening and C. O. Kappe, "Heterogeneous versus Homogeneous Palladium Catalysts for Ligandless Mizoroki-Heck Reactions: A Comparison of Batch/Microwave and Continuous-Flow Processing," Chemistry - A European Journal, Vol. 15, No. 4, 2009, pp. 1001-1010. doi:10.1002/chem.200802200

[52] G. Lu, R. Franzen, Q. Zhang and Y. Xu, "Palladium Charcoal-Catalyzed, Ligandless Suzuki Reaction by Using Tetraarylborates in Water," Tetrahedron Letters, Vol. 46, No. 24, 2005, pp. 4255-4259. doi:10.1016/j.tetlet.2005.04.022

[53] G. Fan, B. Zou, S. Cheng and L. Zheng, "Ligandless Palladium Supported on $\mathrm{SiO}_{2}-\mathrm{TiO}_{2}$ as Effective Catalyst for Suzuki Reaction," Journal of Industrial and Engineering Chemistry, Vol. 16, No. 2, 2010, pp. 220-223. doi:10.1016/j.jiec.2009.08.009

[54] S. P. Stanforth, "Catalytic Cross-Coupling Reactions in Biaryl Synthesis," Tetrahedron, Vol. 54, No. 3-4, 1998, pp. 263-303. doi:10.1016/S0040-4020(97)10233-2

[55] F. Ullmann, "Ueber eine neue Darstellungsweise von Phenyläthersalicylsäure," Berichte der Deutschen Chemischen Gesellschaft, Vol. 37, No. 1, 1904, pp. 853-854.

[56] J. J. Li and G. W. Gribble, "Palladium in Heterocyclic Chemistry," Pergamon, New York, 2000.

[57] C. N. Johnson, G. Stemp, N. Anand, S. C. Stephen and T. Gallagher, "Palladium(0)-catalysed Arylations Using Pyrrole and Indole-2-boronic Acids," Synlett, No. 9, 1998, pp. 1025-1027. doi:10.1055/s-1998-1834

[58] R. J. Sundberg, "Indoles," Academic Press, London, 1996.

[59] A. Zoltewicz and M. P. Cruskie Jr., "Strategies for the Synthesis of Unsymmetrical Quaterpyridines Using Palladium-Catalyzed Cross-Coupling Reactions," Tetrahedron, Vol. 51, No. 42, 1995, pp. 11393-11400. doi:10.1016/0040-4020(95)00699-9

[60] J. Li and W. S. Yue, "Synthesis of 3-Aryl and 3-Heterocyclic Quinoxalin-2-ylamines via Pd-Catalyzed CrossCoupling Reactions," Tetrahedron Letters, Vol. 40, No. 24, 1999, pp. 4507-4510. doi:10.1016/S0040-4039(99)00822-9

[61] T. Soos, G. Timari and G. Hajos, "A New and Concise Synthesis of Furostifoline," Tetrahedron Letters, Vol. 40, No. 49,1999 , pp. 8607-8609. doi:10.1016/S0040-4039(99)01803-1

[62] S. L. Hargreaves, B. L. Pilkington, S. E. Russell and P. A. Worthington, "The Synthesis of Substituted Pyridylpyrimidine Fungicides Using Palladium-Catalysed CrossCoupling Reactions," Tetrahedron Letters, Vol. 41, No. 10, 2000, pp. 1653-1656. doi:10.1016/S0040-4039(00)00007-1

[63] D. Wang and J. Haseltine, "A Comparison of Phenylboronic Acid and Phenyltrimethyltin in the Palladium-Cata- 
lyzed Arylation of 1,5-Dialkylimidazoles," Journal of Heterocyclic Chemistry, Vol. 31, No. 6, 1994, pp. 16371639. doi: $10.1002 /$ ihet.5570310660

[64] D. Peters, A.-B. Hörnfeldt and S. Gronovitz, "Synthesis of Various 5-Substituted Uracils," Journal of Heterocyclic Chemistry, Vol. 27, No. 7, 1990, pp. 2165-2173. doi:10.1002/jhet.5570270756

[65] R. Franzén and J. Tois, "Purine and Sugar Chemistry on Solid Phase-100 Years after the Emil Fischer's Chemistry Nobel Prize 1902," Combinatorial Chemistry \& High Throughput Screening, Vol. 6, No. 5, 2003, pp. 433-434. doi:10.2174/138620703106298617

[66] T. I. Wallow and B. M. Novak, "In Aqua Synthesis of Water-Soluble Poly(para-phenylene) Derivatives," Journal of the American Chemical Society, Vol. 113, No. 19, 1991, pp. 7411-7412. doi:10.1021/ja00019a042

[67] N. G. Andersen, S. P. Maddaford and B. A. Keay, "A Modified in Situ Suzuki Cross-Coupling of Haloarenes for the Preparation of $\mathrm{C}_{2}$ Symmetric Biaryls," Journal of Organic Chemistry, Vol. 61, No. 26, 1996, pp. 9556-9559. doi:10.1021/j09617880

[68] J. Yin and S. L. Buchwald, "A Catalytic Asymmetric Suzuki Coupling for the Synthesis of Axially Chiral Biaryl Compounds," Journal of the American Chemical Society, Vol. 122, No. 48, 2000, pp. 12051-12052. doi: $10.1021 / \mathrm{ja} 005622 \mathrm{z}$

[69] A. N. Cammidge and K. V. L. Crepy, "The First Asymmetric Suzuki Reaction," Chemical Communications, Vol. 18, 2000, pp. 1723-1724. doi:10.1039/b004513f

[70] A.-S. Castanet, F. Colobert, P.-E. Broutin and M. Obringer, "Asymmetric Suzuki Cross-Coupling Reaction: Chirality Reversal Depending on the Palladium-Chiral Phosphine Ratio," Tetrahedron: Asymmetry, Vol. 13, No. 6, 2002, pp. 659-665. doi:10.1016/S0957-4166(02)00169-6

[71] A. Herrbach, A. Marinetti, O. Baudoin, D. Guenard and F. Gueritte, "Asymmetric Synthesis of an Axially Chiral Antimitotic Biaryl via an Atropo-Enantioselective Suzuki Cross-Coupling," Journal of Organic Chemistry, Vol. 68, No. 12, 2003, pp. 4897-4905. doi:10.1021/jo034298y

[72] M. Sevignon, J. Hassan, C. Gozzi, E. Schulz and M. Lemaire, "A New Green Catalytic Method for Biaryl CrossCoupling and Oligothiophene Synthesis," Comptes Rendus de l'Académie des Sciences-Series IIC-Chemistry, Vol. 3, No. 7, 2000, pp. 569-572.

[73] I. Osaka and R. D. McCullough, "Advances in Molecular Design and Synthesis of Regioregular Polythiophenes," Accounts of Chemical Research, Vol. 41, No. 9, 2008, pp. 1202-1214. doi:10.1021/ar800130s

[74] G. Saini, N. T. Lucas and J. Jacob, "Tetrathiophenes with Thiophene Side Chains: Effect of Substitution on Packing and Conjugation," Tetrahedron Letters, Vol. 51, No. 22, 2010, pp. 2956-2958. doi:10.1016/j.tetlet.2010.03.087
[75] N. Metri, X. Sallenave, L. Beouch, C. Plesse, F. Goubard and C. Chevrot, "New Star-Shaped Molecules Derived from Thieno[3,2-b]thiophene Unit and Triphenylamine," Tetrahedron Letters, Vol. 51, No. 50, 2010, pp. 66736676. doi:10.1016/j.tetlet.2010.10.082

[76] R. H. Friend, R. W. Gymer, A. B. Holmes, J. H. Burroughes, R. N. Marks, C. Taliani, D. D. C. Bradley, D. A. dos Santos, M. Logdlund and W. R. Salaneck, "Electroluminescence in Conjugated Polymers," Nature, Vol. 397, 1999, pp. 121-128. doi:10.1038/16393

[77] D. Fichou, "Handbook of Oligo- and Polythiophene," Wiley-VCH, Weinheim, 1998. doi:10.1002/9783527611713

[78] Z. Bao, A. Lovinger and J. Brown, "New Air-Stable n-Channel Organic Thin Film Transistors," Journal of the American Chemistry Society, Vol. 120, No. 1, 1998, pp. 207-208. doi:10.1021/ja9727629

[79] D. B. A. Rep, R. Roelfsema, J. H. van Esch, F. S. Schoonbeek, R. M. Kellogg, B. L. Feringa, T. T. M. Palstra and T. M. Klapwijk, "Self-Assembly of Low-Dimensional Arrays of Thiophene Oligomers from Solution on Solid Substrates," Advanced Materials, Vol. 12, No. 8, 2000, pp. 563-566. doi:10.1002/(SICI)1521-4095(200004)12:8<563::AID-A DMA563>3.0.CO;2-7

[80] F. Garnier, A. Yasser, R. Hajlaoui, G. Horowitz, F. Deloffre, B. Servet, S. Ries and P. Alnot, "Molecular Engineering of Organic Semiconductors: Design of Self-Assembly Properties in Conjugated Thiophene Oligomers," Journal of the American Chemistry Society, Vol. 115, No. 19, 1993, pp. 8716-8721. doi:10.1021/ja00072a026

[81] R. D. McCullough, R. D. Lowe, M. Jayaraman, P. C. Ewbank, D. L. Anderson and S. Tristram-Nagle, "Novel Coordination Complexes of Tetrathiafulvalene Derivatives," Synthetic Metals, Vol. 55, No. 1, 1993, pp. 1198 1203.

[82] K. Faid, R. Cloutier and M. Leclerc, "Design of Novel Electroactive Polybithiophene Derivatives," Macromolecules, Vol. 26, No. 10, 1993, pp. 2501-2507. doi: $10.1021 / \mathrm{ma} 00062 \mathrm{a} 017$

[83] H. Sirringhaus, P. J. Brown, R. H. Friend, M. M. Nielsen, K. Bechgaard, B. M. W. Langeveld-Voss, A. J. H. Spiering, R. A. J. Janssen, E. W. Meijer, P. Herwig and D. M. De Leeuw, "Two-Dimensional Charge Transport in SelfOrganized, High-Mobility Conjugated Polymers," Nature, Vol. 401, 1999, pp. 685-688. doi:10.1038/44359

[84] Y. Zheng, H. Zhou, D. Liu, G. Floudas, M. Wagner, K. Koynov, M. Mezger, H. J. Butt and T. Ikeda, "Supramolecular Thiophene Nanosheets," Angewandte Chemie International Edition, Vol. 52, No. 18, 2013, pp. 4845-4848. doi:10.1002/anie. 201210090 